\title{
A mind is like a parachute
}

\author{
Thoralf M. Sundt, MD
}

\author{
From the Division of Cardiac Surgery, Massachusetts General Hospital, Boston, Mass; and the Department of Sur- \\ gery, Harvard Medical School, Boston, Mass. \\ Disclosures: Author has nothing to disclose with regard to commercial support. \\ Received for publication Oct 22, 2017; accepted for publication Oct 26, 2017. \\ Address for reprints: Thoralf M. Sundt, MD, Division of Cardiac Surgery, Massachusetts General Hospital, 55 \\ Fruit St, Boston, MA 02114 (E-mail: tsundt@mgh.harvard.edu). \\ J Thorac Cardiovasc Surg 2018;155:699 \\ $0022-5223 / \$ 36.00$ \\ Copyright (c) 2017 by The American Association for Thoracic Surgery \\ https://doi.org/10.1016/j.jtcvs.2017.10.130
}

I do not know about you, but "lessons" from aviation trigger an emotional response in me, and not always a positive one. I loved reading about Chuck Yeager, but I find it a bit insulting when someone in a flight suit tells me our problem is that we do not die with the patient the way a pilot goes down with the plane. What does this person know about getting an 88-year-old who is "too sick for transcatheter aortic valve replacement" through an aortic valve replacement in conjunction with coronary artery bypass grafting? Phooey.

But I have to admit that we are still a long way from the 6sigma "high reliability" discussed in the article by Hickey and colleagues ${ }^{1}$ in this issue of the Journal. We also know that just trying harder is not enough. Mistakes are still made, errors still occur, and patients still get hurt.

What can we do? What do we do when we are unhappy with the technical aspects of an operation? We see how others have solved the problem that we face, and we learn. This is how we iteratively improve as technical surgeons. An inclusion wrap was an entirely acceptable approach to root replacement in the past, but late pseudoaneurysms pressed for improvement. The button technique was developed by a few and then adopted by the many. Can we say the same for error management? Are we open to learning how others have addressed this problem?

Although I am skeptical of the immediate "off the shelf" application of aviation solutions to medicine, the essence of the Threat and Error model rings quite true. In identifying an "unintended state" as a threat to safety, a name is put to something that I suspect we all do in an intuitive way: when a case with a resident is drifting off plan and into "the danger zone," I move to the right side of the table. Labeling this state helps us to think more about its implications. This is where it gets interesting.

The first step after identifying the unintended state is to "prioritize maximizing safety margin" before jumping in to solve the problem. I had not thought about it that way before, but again it rings true. "Stop and take your own pulse first," and then be sure you have marshaled all your

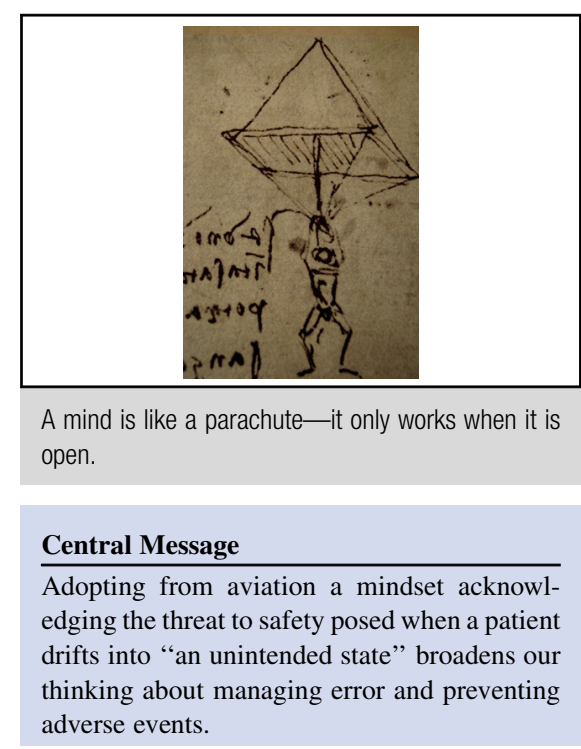

See Article page 690.

resources. Call for help. Engage the team. Then dive into the solution.

And there is more. The focus on the unintended state as a threat in and of itself heightens our awareness and broadens our thinking about error management in the same way that attention to near misses in addition to adverse events did so. Recognition that a patient in an unintended state is more vulnerable to a "chain of errors" is reminiscent of the admonition, "It is not the first mistake that kills the patient, it is the second." This is a call for increased vigilance and for getting us back on track! The practice of "performance rounds," with identification of episodes during which any patient is in an unintended state, institutionalizes this thinking.

Where does that leave us? Are aviation solutions applicable to what we do when what we do is so much more complex? I am not sure. I do think that aviation has shown us that a formal approach to error management can improve both system and individual performance in a complex task. If what we do is really that much more complex, does that not argue that we need such an approach even more? Let's keep an open mind-even to aviation.

\section{Reference}

1. Hickey EJ, Halvorsen F, Laussen PC, Hirst G, Schwartz S, Van Arsdell GS Chasing the 6-sigma: drawing lessons from the cockpit culture. J Thorac Cardiovasc Surg. 2018;155:690-6.e1. 